\title{
ON THE UNIQUE CONTINUATION OF SOLUTIONS TO THE GENERALIZED KDV EQUATION
}

\author{
Carlos E. Kenig, Gustavo Ponce, and Luis Vega
}

\section{Introduction}

In this paper we shall prove that solutions of the generalized Korteweg-de Vries equation are uniquely determined by their values on a semi-line at two different times. In particular, if $u_{j}=u_{j}(x, t), j=1,2$ are real valued solutions of the $k$-generalized Korteweg-de Vries $(k$-gKdV) equation

$$
\partial_{t} u+\partial_{x}^{3} u+u^{k} \partial_{x} u=0, \quad(x, t) \in \mathbb{R} \times\left[t_{1}, t_{2}\right], \quad k \in \mathbb{Z}^{+},
$$

with $t_{1}<t_{2}$ which are sufficiently smooth and such that for some $b \in \mathbb{R}$

$$
u_{1}(x, t)=u_{2}(x, t), \quad(x, t) \in(b, \infty) \times\left\{t_{1}, t_{2}\right\}\left(\text { or }(-\infty, b) \times\left\{t_{1}, t_{2}\right\}\right),
$$

then $u_{1} \equiv u_{2}$.

For $k=1$ the equation (1.1) was derived by Korteweg-de Vries [9] as a model for long waves propagating in a channel. Subsequently the KdV and its generalized form have been shown to be relevant in several physical situations, see references in [3].

In [13] B. Zhang proved the above result for the case of the KdV equation

$$
\partial_{t} u+\partial_{x}^{3} u+u \partial_{x} u=0
$$

and for the equation

$$
\partial_{t} u+\partial_{x}^{3} u-u^{2} \partial_{x} u=0
$$

Received September 25, 2003.

2000 Mathematics Subject Classification. Primary 35Q53; secondary 35G25, 35D99.

C. E. K. and G. P. were supported by NSF grants. L. V. was supported by a DGICYT grant.

Key words and phrases. Korteweg-de Vries equation, compact support, Carleman estimates 
under the assumption that $u_{2} \equiv 0$, by using inverse scattering theory and Miura's transformation. In [5] we established the above result for very general Kortewegde Vries type of equations again under the restriction $u_{2} \equiv 0$.

In [6] we obtained the corresponding result for the semilinear Schrödinger equation

$$
i \partial_{t} u=\Delta u+F(u, \bar{u}), \quad t \in \mathbb{R}, x \in \mathbb{R}^{n},
$$

in dimension $n \geq 1$, for any pair of solutions $u_{1}, u_{2}$, under very general assumptions on the nonlinearity $F$ and the class of solutions considered.

The main problem in extending the results in [5], [13] to any pair of solutions $u_{1}, u_{2}$ is that one needs to consider the equation for the difference $u_{1}-u_{2}$, which does not have the symmetry of the original equation which was needed to apply the arguments there. This seems to be more evident in the case of inverse scattering theory. Also the arguments in [6] do not apply here since the equation for the difference is not semilinear, it involves gradient terms. These terms cannot be handled by the approach in [6]. At the linear level this translates into establishing unique continuation properties for first order perturbations of the Airy equation. The case where second order perturbation terms are considered reduces to this one by an appropriate change of variable.

In this work we shall extend the method of proof used in [5] to treat the equation for the difference of solutions of (1.1) instead of the equation (1.1) itself.

The statement of our main result is the following.

Theorem 1. Let $u_{j}, j=1,2$ be sufficiently smooth (see (1.8)-(1.10) below) real valued solutions of the equation

$$
\partial_{t} u+\partial_{x}^{3} u+F\left(x, t, u, \partial_{x} u, \partial_{x}^{2} u\right)=0, \quad(x, t) \in \mathbb{R} \times\left[t_{1}, t_{2}\right],
$$

where $F \in C_{b}^{8}$ in $(x, t)$, of polynomial growth in the other variables, at least quadratic in $u, \partial_{x} u, \partial_{x}^{2} u$ in the terms involving $\partial_{x}^{2} u$, i.e. $\partial F(x, t, 0,0,0) / \partial x_{5}=0$, for any $(x, t) \in \mathbb{R} \times\left[t_{1}, t_{2}\right]$.

Assume that $u_{j}, j=1,2$, have some decay (see (1.8) below) if $F$ is just quadratic in $u, \partial_{x} u, \partial_{x}^{2} u$ in the terms involving $\partial_{x}^{2} u$.

If there exists $b \in \mathbb{R}$ such that

$$
\begin{aligned}
u_{1}(x, t) & =u_{2}(x, t), \quad(x, t) \in(b, \infty) \times\left\{t_{1}, t_{2}\right\} \\
\text { or } \quad u_{1}(x, t) & =u_{2}(x, t), \quad(x, t) \in(-\infty, b) \times\left\{t_{1}, t_{2}\right\},
\end{aligned}
$$

then $u_{1} \equiv u_{2}$.

Remark: (a) Concerning the regularity hypothesis on the solutions $u_{j}$ 's required in Theorem 1 it suffices to assume that

$$
u_{j} \in C\left(\left[t_{1}, t_{2}\right]: H^{6}(\mathbb{R}) \cap L^{2}\left(|x|^{6} d x\right)\right) \cap C^{1}\left(\left[t_{1}, t_{2}\right]: H^{3}(\mathbb{R})\right), \quad j=1,2,
$$


for the general case and

$$
u_{j} \in C\left(\left[t_{1}, t_{2}\right]: H^{7}(\mathbb{R})\right) \cap C^{1}\left(\left[t_{1}, t_{2}\right]: H^{4}(\mathbb{R})\right), \quad j=1,2,
$$

if the nonlinearity $F$ is at least cubic in $u, \partial_{x} u, \partial_{x}^{2} u$ in the terms involving $\partial_{x}^{2} u$, see [4].

(b) To simplify the exposition we will carry out the details only in the case of the $k$-gKdV equation (1.1), and explain the necessary modifications to treat the general equation in (1.6). In the case of the $k$-gKdV equation it suffices to assume that

$$
u_{j} \in C\left(\left[t_{1}, t_{2}\right]: H^{4}(\mathbb{R})\right) \cap C^{1}\left(\left[t_{1}, t_{2}\right]: H^{1}(\mathbb{R})\right), \quad j=1,2 .
$$

For the existence theory we refer to [3].

(c) The proof of Theorem 1 consists of three main steps. First we establish appropriate weighted (exponential) energy estimates for the solution of the equation satisfied for the difference of the two solutions $w(x, t)=u_{1}(x, t)-u_{2}(x, t)$. In the second step we prove some Carleman estimates of the type established by Kenig-Ruiz-Sogge [7] and Kenig-Sogge [8]. As in [7], [8], the estimate used in [5] is related to the so called Strichartz estimates involving the $L_{t}^{q} L_{x}^{p}$-norm. Here we need a new estimate (Lemma 2.2 estimate (2.8)) which is related to the smoothing effect found in [2] and [10] which describes a gain of derivatives in the $L_{x}^{p} L_{t}^{q}$-norm (i.e. first the norm in the $t$-variable then in the $x$-variable). This gain of derivatives is essential in our argument. In the final step we show that the problem reduces to one where the local unique continuation principle obtained by Saut and Scheurer in [11] can be applied.

(d) We observe that no analyticity assumptions on the nonlinearity $F$ are required.

The paper is organized as follows. In Section 2 we prove Theorem 1 for the $k$-generalized KdV equation (1.1). In Section 3 we sketch the necessary modifications to treat the general case.

\section{Proof of Theorem 1 in the case of the $k$-generalized $\mathrm{KdV}$ equation}

We consider solutions $u_{j}, j=1,2$, of the $k$-generalized KdV equation (1.1) with the regularity described in (1.10). Also without loss of generality we assume that $t_{1}=0, t_{2}=1$ and that

$$
u_{1}(x, t)=u_{2}(x, t), \quad(x, t) \in(b, \infty) \times\left\{t_{1}, t_{2}\right\} .
$$

We need some preliminary results. The first one is concerned with the decay properties of solutions to a $k$-gKdV type of equation. 
Lemma 2.1. Let $j \in \mathbb{Z}, j \geq 1$. Let $\omega \in C\left([0,1]: H^{j+1}(\mathbb{R})\right)$ be a solution of the linear initial value problem

$$
\left\{\begin{array}{l}
\partial_{t} \omega+\partial_{x}^{3} \omega+a_{1}(x, t) \partial_{x} \omega+a_{2}(x, t) \omega=0, \quad t \in[0,1], x \in \mathbb{R} \\
\omega(x, 0)=\omega_{0}(x)
\end{array}\right.
$$

where the real coefficients $a_{1} \in C\left([0,1]: H^{j+1}(\mathbb{R})\right), a_{2} \in C\left([0,1]: H^{j}(\mathbb{R})\right)$ and such that for a given $\beta>0$

$$
e^{\beta x} \omega_{0}, . ., e^{\beta x} \partial_{x}^{j} \omega_{0} \in L^{2}(\mathbb{R})
$$

Then

$$
\begin{aligned}
& \sup _{t \in[0,1]}\left\|e^{\beta x} \omega(t)\right\|_{C^{j-1}} \\
& \leq c\left(k ; \beta ;\left(\left\|e^{\beta x} \partial_{x}^{l} \omega_{0}\right\|_{L^{2}}\right)_{l=0}^{j} ; \sup _{t \in[0,1]}\left(\left\|a_{1}(t)\right\|_{H^{j+1}}+\left\|a_{2}(t)\right\|_{H^{j}}\right)\right) .
\end{aligned}
$$

The proof of Lemma 2.1 is similar to that given in [2], [5] for the $k$-gKdV equation.

To state the next results we need to introduce some notation,

$$
f=f(x, t) \in C^{3,1}\left(\mathbb{R}^{2}\right) \quad \text { if } \quad \partial_{x} f, \partial_{x}^{2} f, \partial_{x}^{3} f, \partial_{t} f \in C\left(\mathbb{R}^{2}\right),
$$

and

$$
f=f(x, t) \in C_{0}^{3,1}\left(\mathbb{R}^{2}\right) \quad \text { if } \quad f \in C^{3,1}\left(\mathbb{R}^{2}\right) \text { and has compact support. }
$$

Next, following the ideas in Kenig-Ruiz-Sogge [7] and Kenig-Sogge [8] we have the following Carleman estimates.

Lemma 2.2. If $f \in C_{0}^{3,1}\left(\mathbb{R}^{2}\right)$ (see (2.5)-(2.6)), then

$$
\left\|e^{\lambda x} f\right\|_{L^{8}\left(\mathbb{R}^{2}\right)} \leq c_{0}\left\|e^{\lambda x}\left\{\partial_{t}+\partial_{x}^{3}\right\} f\right\|_{L^{8 / 7}\left(\mathbb{R}^{2}\right)},
$$

and

$$
\left\|e^{\lambda x} \partial_{x} f\right\|_{L_{x}^{16} L_{t}^{16 / 5}\left(\mathbb{R}^{2}\right)} \leq c_{0}\left\|e^{\lambda x}\left\{\partial_{t}+\partial_{x}^{3}\right\} f\right\|_{L_{x}^{16 / 15} L_{t}^{16 / 11}\left(\mathbb{R}^{2}\right)},
$$

for all $\lambda \geq 0$, with $c_{0}$ independent of $\lambda$ and the support of $f$.

Proof of Lemma 2.2.

For the proof of (2.7) we refer to [5] (Lemma 2.3).

To prove $(2.8)$ we first observe that by rescaling

$$
h_{\lambda}(x, t)=f\left(\lambda x, \lambda^{3} t\right) \text {, }
$$


we have

$$
\left\|e^{\lambda x} \partial_{x} h_{\lambda}\right\|_{L_{x}^{16} L_{t}^{16 / 5}}=\lambda^{1-1 / 16-15 / 16}\left\|e^{x} \partial_{x} f\right\|_{L_{x}^{16} L_{t}^{16 / 5}},
$$

and

$$
\begin{aligned}
& \left\|e^{\lambda x}\left\{\partial_{t}+\partial_{x}^{3}\right\} h_{\lambda}\right\|_{L_{x}^{16 / 15} L_{t}^{16 / 11}} \\
& =\lambda^{3-15 / 16-33 / 16}\left\|e^{x}\left\{\partial_{t}+\partial_{x}^{3}\right\} f\right\|_{L_{x}^{16 / 15} L_{t}^{16 / 11}}
\end{aligned}
$$

So it suffices to prove $(2.8)$ for $\lambda=1$, i.e.

$$
\left\|e^{x} \partial_{x} f\right\|_{L_{x}^{16} L_{t}^{16 / 5}} \leq c\left\|e^{x}\left\{\partial_{t}+\partial_{x}^{3}\right\} f\right\|_{L_{x}^{16 / 15} L_{t}^{16 / 11}},
$$

since the case $\lambda=0$ follows by taking the limit as $\lambda \downarrow 0$ in both sides of (2.8).

Defining

$$
g(x, t)=e^{x} f(x, t), \quad f(x, t)=e^{-x} g(x, t),
$$

and using that

$$
e^{x} \partial_{x}\left(e^{-x} g\right)=\left(\partial_{x}-1\right) g
$$

and

$$
e^{x}\left\{\partial_{t}+\partial_{x}^{3}\right\}\left(e^{-x} g\right)=\left\{\partial_{t}+\partial_{x}^{3}-3 \partial_{x}^{2}+3 \partial_{x}-1\right\} g,
$$

we find that $(2.12)$ is equivalent to

$$
\left\|\left(\partial_{x}-1\right) g\right\|_{L_{x}^{16} L_{t}^{16 / 5}} \leq c\left\|\left\{\partial_{t}+\partial_{x}^{3}-3 \partial_{x}^{2}+3 \partial_{x}-1\right\} g\right\|_{L_{x}^{16 / 15} L_{t}^{16 / 11}},
$$

for $g \in C_{0}^{3,1}\left(\mathbb{R}^{2}\right)$. Taking Fourier transform in both variables $(x, t)$ and defining

$$
h(x, t)=\left\{\partial_{t}+\partial_{x}^{3}-3 \partial_{x}^{2}+3 \partial_{x}-1\right\} g(x, t), \quad \hat{g}(\xi, \tau)=\frac{\hat{h}(\xi, \tau)}{i\left(\tau-(\xi+i)^{3}\right)}
$$

with $\hat{h}(\xi, \tau)$ vanishing near the zeros of $P(\xi, \tau)=i\left(\tau-(\xi+i)^{3}\right)$, we rewrite (2.16) as the following multiplier estimate

$$
\left\|T_{m_{0}} h\right\|_{L_{x}^{16} L_{t}^{16 / 5}}=\left\|\left(m_{0}(\xi, \tau) \hat{h}(\xi, \tau)\right)^{\vee}\right\|_{L_{x}^{16} L_{t}^{16 / 5}} \leq c\|h\|_{L_{x}^{16 / 15} L_{t}^{16 / 11}},
$$

for functions $h \in \mathcal{S}\left(\mathbb{R}^{2}\right)$, whose Fourier transform is 0 near the zeros of the denominator (up to a multiplicative constant)

$$
d(\xi, \tau)=\tau-(\xi+i)^{3},
$$


of the multiplier

$$
m_{0}(\xi, \tau)=\frac{i \xi-1}{i\left(\tau-(\xi+i)^{3}\right)}
$$

To obtain (2.18) we first consider the multiplier

$$
m_{1}(\xi, \tau)=\frac{(i \xi-1) \xi}{i\left(\tau-(\xi+i)^{3}\right)}
$$

For $|\tau| \geq 1 / 2$ we write

$$
\begin{gathered}
m_{1}(\xi, \tau)=i\left(\frac{i \xi^{2}-\xi}{(\xi+i)^{3}-\tau}\right)=i\left(\frac{A}{\xi-a}+\frac{B}{\xi-b}+\frac{C}{\xi-c}\right) . \\
(\xi-a)(\xi-b)(\xi-c)=(\xi+i)^{3}-\tau
\end{gathered}
$$

thus

$$
\left\{\begin{array}{l}
a b c=i+\tau \\
a b+b c+a c=-3 \\
a+b+c=-3 i
\end{array}\right.
$$

and

$$
M\left(\begin{array}{l}
A \\
B \\
C
\end{array}\right)=\left(\begin{array}{ccc}
1 & 1 & 1 \\
b+c & a+c & a+b \\
b c & a c & a b
\end{array}\right)\left(\begin{array}{l}
A \\
B \\
C
\end{array}\right)=\left(\begin{array}{l}
i \\
1 \\
0
\end{array}\right)
$$

with

$$
\operatorname{det} M=a^{2}(b-c)+b^{2}(c-a)+c^{2}(a-b)=(c-b)(b-a)(a-c) .
$$

Notice that

(i) If we perform a permutation of $\{a, b, c\}$, the resulting matrix is obtained from $M$ by a permutation of the columns of $M$.

(ii) If $a<b<c$, then $\operatorname{det} M \neq 0$.

(iii) If $a=b$, then $\operatorname{det} M=0$.

(iv) For $\tau \in \mathbb{R}$ with $|\tau| \geq 1 / 4$ the denominator $d(\xi, \tau)=\tau-(\xi+i)^{3}$ of the multipliers $m_{0}, m_{1}$ (see (2.20)-(2.21)) as a function of $\xi$ does not have a double zero.

(v) Changing variables

$$
a=\tau^{1 / 3} a_{1}, \quad b=\tau^{1 / 3} b_{1}, \quad c=\tau^{1 / 3} c_{1},
$$


(2.24) becomes

$$
\left\{\begin{array}{l}
a_{1} b_{1} c_{1}=i / \tau+1 \\
a_{1} b_{1}+b_{1} c_{1}+a_{1} c_{1}=-3 / \tau^{2 / 3} \\
a_{1}+b_{1}+c_{1}=-3 i / \tau^{1 / 3}
\end{array}\right.
$$

with $a_{1}=a_{1}(\tau), b_{1}=b_{1}(\tau), c_{1}=c_{1}(\tau)$.

Claim: If $|\tau| \geq 1 / 2$, then $a_{1}, b_{1}, c_{1}$ are bounded.

Proof of the claim

If $\left|a_{1}\right|>K$ with $K>>1$ from (2.28) it follows that $\left|b_{1} c_{1}\right| \leq 3 / K$, so by the second equation one has $\left|a_{1}\left(b_{1}+c_{1}\right)\right| \leq 3 / K+3 / \tau^{2 / 3}$ which implies that $\left|b_{1}+c_{1}\right| \leq 3 / K^{2}+3 / K \tau^{2 / 3} \leq 3 / K^{2}+6 / K$. Finally using the last equation in (2.28) we get

$$
3 / K^{2}+6 / K+6>\left|b_{1}+c_{1}+3 i / \tau^{1 / 3}\right|=\left|a_{1}\right|>K,
$$

which is a contradiction. This proves the claim.

Next we shall find a lower bound for $\operatorname{det} M_{1}$ where

$$
\operatorname{det} M=\tau\left(c_{1}-b_{1}\right)\left(b_{1}-a_{1}\right)\left(a_{1}-c_{1}\right)=\tau \operatorname{det} M_{1}, \quad \text { for }|\tau| \geq 1 / 4
$$

To show this we differentiate both sides in (2.23) and evaluate the result in $a, b, c$ to get

$$
\begin{aligned}
& (a-b)(a-c)=3(a+i)^{2}, \\
& (b-a)(b-c)=3(b+i)^{2}, \\
& (c-a)(c-b)=3(c+i)^{2},
\end{aligned}
$$

respectively. Multiplying the identities in (2.31) one sees that

$$
-(c-b)^{2}(b-a)^{2}(a-c)^{2}=27(a+i)^{2}(b+i)^{2}(c+i)^{2} .
$$

Combining (2.26), (2.32), and (2.23) it follows that

$$
(\operatorname{det} M)^{2}=-27 \tau^{2}
$$

Hence,

$$
|\operatorname{det} M| \geq 5|\tau|,
$$

and by (2.30) for any $\tau \in \mathbb{R}$

$$
\left|\operatorname{det} M_{1}\right| \geq 5 \text {. }
$$


Thus, if $|\tau| \geq 1 / 4$ we have that $a_{1}, b_{1}, c_{1}$ are bounded and $\operatorname{det} M_{1}$ is bounded below. Since

$$
M=\left(\begin{array}{ccc}
1 & 0 & 0 \\
0 & \tau^{1 / 3} & 0 \\
0 & 0 & \tau^{2 / 3}
\end{array}\right) M_{1}, \quad M^{-1}=M_{1}^{-1}\left(\begin{array}{ccc}
1 & 0 & 0 \\
0 & \tau^{-1 / 3} & 0 \\
0 & 0 & \tau^{-2 / 3}
\end{array}\right)
$$

and

$$
\left(\begin{array}{l}
A \\
B \\
C
\end{array}\right)=M^{-1}\left(\begin{array}{l}
i \\
1 \\
0
\end{array}\right)
$$

Returning to $(2.22)$ we can write

$$
\begin{aligned}
& \frac{A}{\xi-a}=\frac{A(\tau)}{\xi-\tau^{1 / 3} a_{1}(\tau)} \\
& =\frac{A(\tau)}{\xi-\tau^{1 / 3}\left(\operatorname{Re} a_{1}(\tau)+i \operatorname{Im} a_{1}(\tau)\right)},
\end{aligned}
$$

with $A(\tau)$ and $a_{1}(\tau)$ both bounded for $|\tau| \geq 1 / 4$. For $\tau^{1 / 3} \operatorname{Im} a_{1}(\tau)>0$ one has

$$
\frac{1}{\xi-i \tau^{1 / 3} \operatorname{Im} a_{1}(\tau)}=c_{0}\left(\chi_{(0, \infty)}(x) e^{-\tau^{1 / 3} \operatorname{Im} a_{1}(\tau) x}\right)^{\wedge}
$$

with a similar result for the case $\tau^{1 / 3} \operatorname{Im} a_{1}(\tau)<0$. The other fractions in $(2.22)$

$$
\frac{B(\tau)}{\xi-b(\tau)}, \quad \frac{C(\tau)}{\xi-c(\tau)},
$$

can be treated in the same manner. Hence, it follows that for $|\tau| \geq 1 / 4$

$$
\begin{aligned}
& i\left(\frac{A}{\xi-a}+\frac{B}{\xi-b}+\frac{C}{\xi-c}\right)^{\vee_{\xi}}(x) \\
& =\left(m_{1}(\cdot, \tau)\right)^{\vee_{\xi}}(x)=K(x, \tau) \in L^{\infty}(\mathbb{R} \times\{|\tau| \geq 1 / 4\}) .
\end{aligned}
$$

For the case $|\tau| \leq 1 / 4$ we observe that for $|\xi| \geq 4$ one has that

$$
\left|\frac{(i \xi-1) \xi}{i\left(\tau-(\xi+i)^{3}\right)}+\frac{1}{\xi}\right| \leq \frac{c}{\xi^{2}}
$$

with $c$ uniform for $|\tau| \leq 1 / 2$. Finally, if $|\tau| \leq 1 / 4$ and $|\xi| \leq 4$ we use that $P(x, \xi)=i\left(\tau-(\xi+i)^{3}\right)$ does not vanish, so that

$$
\frac{(i \xi-1) \xi}{i\left(\tau-(\xi+i)^{3}\right)} \in C^{\infty}
$$


and the desired bound is straightforward.

Collecting the information in (2.41)-(2.43), a simple argument shows that (see $[3])$,

$$
\left\|T_{m_{1}} h\right\|_{L_{x}^{\infty} L_{t}^{2}}=\left\|\left(m_{1}(\xi, \tau) \hat{h}(\xi)\right)^{\vee}\right\|_{L_{x}^{\infty} L_{t}^{2}} \leq c\|h\|_{L_{x}^{1} L_{t}^{2}} .
$$

From (2.20) and (2.21) one has that

$$
T_{m_{1}} h=\left(\partial_{x}-1\right) \partial_{x} T_{d^{-1}} h, \quad T_{m_{0}} h=\left(\partial_{x}-1\right) T_{d^{-1}} h .
$$

Next we shall perform a Littlewood-Paley decomposition. Hence, consider the sequence $\Gamma=\left\{\varphi_{j}: j=0,1,2, \ldots\right\}$ where $\varphi_{j} \geq 0, \varphi_{j} \in C_{0}^{\infty}(\mathbb{R}), j=0,1,2, \ldots$ with $\operatorname{supp} \varphi_{j} \subset\left(-2^{j},-2^{j-1}\right) \cup\left(2^{j-1}, 2^{j}\right), j=1,2, .$. , supp $\varphi_{0} \subset(-2,2)$ and $\sum_{j=0}^{\infty} \varphi_{j}=1$ (also the $\tilde{\varphi}_{j}$ 's denote an appropriate modified version of $\Gamma$ ) we have

$$
\begin{aligned}
& 2^{-j}\left\|\left(\partial_{x}-1\right) \partial_{x} T_{d^{-1}}\left(\varphi_{j} \hat{h}\right)^{\vee}\right\|_{L_{x}^{\infty} L_{t}^{2}} \\
& \leq c 2^{-j}\left\|\left(\varphi_{j} \hat{h}\right)^{\vee}\right\|_{L_{x}^{1} L_{t}^{2}}, \quad j=0,1,2, \ldots
\end{aligned}
$$

Hence, using the notation

$$
T_{j}(h)=T_{d^{-1}}\left(\varphi_{j} \hat{h}\right)^{\vee}, \quad \tilde{T}_{j}(h)=T_{d^{-1}}\left(\tilde{\varphi}_{j} \hat{h}\right)^{\vee},
$$

it follows that

$$
\begin{aligned}
& \left\|\left(\partial_{x}-1\right) T_{j}(h)\right\|_{L_{x}^{\infty} L_{t}^{2}} \leq c 2^{-j}\left\|\left(\partial_{x}-1\right) \partial_{x} \tilde{T}_{j}(h)\right\|_{L_{x}^{\infty} L_{t}^{2}} \\
& \leq c 2^{-j}\|h\|_{L_{x}^{1} L_{t}^{2}}, \quad j=0,1,2, \ldots
\end{aligned}
$$

and by (2.7) and its proof (see [5], Lemma 2.3)

$$
\left\|\left(\partial_{x}-1\right) T_{j}(h)\right\|_{L_{x}^{8} L_{t}^{8}} \leq 2^{j}\left\|T_{j}(h)\right\|_{L_{x}^{8} L_{t}^{8}} \leq 2^{j}\|h\|_{L_{x}^{8 / 7} L_{t}^{8 / 7}}, j=0,1,2, . .
$$

by interpolation (see [12]) one gets that

$$
\left\|\left(\partial_{x}-1\right) T_{j}(h)\right\|_{L_{x}^{16} L_{t}^{16 / 5}} \leq c\|h\|_{L_{x}^{16 / 15} L_{t}^{16 / 11}}
$$

and since $\left(\partial_{x}-1\right) T_{j}(h)=\left(\partial_{x}-1\right) T_{j}\left(\tilde{\varphi}_{j} \hat{h}\right)^{\vee}$, using Littlewood-Paley theory

$$
\begin{aligned}
& \left\|T_{m_{0}}(h)\right\|_{L_{x}^{16} L_{t}^{16 / 5}}=\left\|\left(\partial_{x}-1\right) T_{d^{-1}}(h)\right\|_{L_{x}^{16} L_{t}^{16 / 5}} \\
& \left.\leq\left\|\left(\sum_{j=0}^{\infty}\left|\left(\partial_{x}-1\right) T_{j}(h)\right|^{2}\right)^{1 / 2}\right\|_{L_{x}^{16} L_{t}^{16 / 5}}\right)^{1 / 2} \\
& \leq\left(\sum_{j=0}^{\infty}\left\|\left(\partial_{x}-1\right) T_{j}(h)\right\|_{L_{x}^{16} L_{t}^{16 / 5}}^{2}\right)^{1 / 2} \leq c\|h\|_{L_{x}^{16 / 15} L_{t}^{16 / 11}},
\end{aligned}
$$

which completes the proof of (2.8). 
Lemma 2.3. The inequalities (2.7)-(2.8) still hold for $g \in C^{3,1}(\mathbb{R} \times[0,1]$ ) (see (2.5)) such that

$$
\sum_{j \leq 2}\left|\partial_{x}^{j} g(x, t)\right| \leq c_{\beta} e^{-\beta|x|}, \quad t \in[0,1], \quad \forall \beta>0
$$

and

$$
g(x, 0)=g(x, 1)=0, \quad \forall x \in \mathbb{R} .
$$

For the proof of Lemma 2.3 we refer to [5] (Lemmas 2.4-2.5).

In the proof of Theorem 1 we also need the following local unique continuation result due to Saut-Scheurer [11].

Theorem 2.4 [11]. Assume that $v=v(x, t)$ satisfies the equation

$$
\partial_{t} v+\partial_{x}^{3} v+\sum_{j=0}^{2} r_{j}(x, t) \partial_{x}^{j} v=0, \quad(x, t) \in(a, b) \times\left(t_{1}, t_{2}\right),
$$

with

$$
r_{j} \in L^{\infty}\left(\left(t_{1}, t_{2}\right): L_{l o c}^{2}((a, b)) .\right.
$$

If $v$ vanishes on an open set $\Omega \subseteq(a, b) \times\left(t_{1}, t_{2}\right)$, then $v$ vanishes in the horizontal components of $\Omega$, i.e. the set

$$
\left\{(x, t) \in(a, b) \times\left(t_{1}, t_{2}\right): \exists y \text { s.t. }(y, t) \in \Omega\right\} .
$$

Proof of Theorem 1.

We define

$$
w(x, t)=u_{1}(x, t)-u_{2}(x, t)
$$

which satisfies the equation

$$
\partial_{t} w+\partial_{x}^{3} w+V_{1}(x, t) \partial_{x} w+V_{2}(x, t) w=0,
$$

with

$$
V_{1}(x, t)=u_{1}^{k}, \quad V_{2}(x, t)=\left(u_{1}^{k-1}+u_{1}^{k-2} u_{2}+\ldots+u_{2}^{k-1}\right) \partial_{x} u_{2} .
$$

Since from (2.1)

$$
\text { supp } w(\cdot, 0), \operatorname{supp} w(\cdot, 1) \subseteq(-\infty, b],
$$


Lemma 2.1 shows that for any $\beta>0$

$$
\sum_{j \leq 2}\left|\partial_{x}^{j} w(x, t)\right| \leq c_{b, \beta} e^{-\beta x}, \quad \text { for } x>0, t \in[0,1] .
$$

We will show that there exists a large number $R>0$ such that

$$
\operatorname{supp} w(\cdot, t) \subseteq(-\infty, 2 R], \quad \forall t \in[0,1],
$$

then Saut-Scheurer's result (Theorem 2.4) will complete the proof.

Let $\mu \in C^{\infty}(\mathbb{R})$ be a nondecreasing function such that $\mu(x)=0, x \leq 1$ and $\mu(x)=1, x \geq 2$. Let $\mu_{R}(x)=\mu(x / R)$ with $R>1$. Define

$$
V_{j}^{R}=V_{j}(x, t) \chi_{\{x \geq R\}}(x) \in L_{x}^{p} L_{t}^{q}(\mathbb{R} \times[0,1]),
$$

for any $p, q \in[1, \infty], j=1,2$, (see (1.10), (2.60), and (2.62)) and

$$
w_{R}(x, t)=\mu_{R}(x) w(x, t) .
$$

Combining our assumptions (1.10) and (2.61)-(2.62) we can apply Lemma 2.3 to $w_{R}(x, t)$ for $R$ sufficiently large. Thus, we have

$$
\begin{aligned}
& \left\{\partial_{t}+\partial_{x}^{3}\right\} w_{R}=\left\{\partial_{t}+\partial_{x}^{3}\right\}\left(\mu_{R} w\right)=-V_{1} \partial_{x}\left(\mu_{R} w\right)-\mu_{R} V_{2} w \\
& +V_{1} \partial_{x} \mu_{R} w+3 \partial_{x} \mu_{R} \partial_{x}^{2} w+3 \partial_{x}^{2} \mu_{R} \partial_{x} w+\partial_{x}^{3} \mu_{R} w \\
& =-V_{1} \partial_{x}\left(\mu_{R} w\right)-V_{2} \mu_{R} w+F_{1}+F_{2}+F_{3}+F_{4} \\
& =-V_{1} \partial_{x}\left(\mu_{R} w\right)-V_{2} \mu_{R} w+F_{R} .
\end{aligned}
$$

Hence, it follows that

$$
\begin{aligned}
& \left\|e^{\lambda x} \mu_{R} w\right\|_{L^{8}(\mathbb{R} \times[0,1])}+\left\|e^{\lambda x} \partial_{x}\left(\mu_{R} w\right)\right\|_{L_{x}^{16} L_{t}^{16 / 5}(\mathbb{R} \times[0,1])} \\
& \leq c_{0}\left\|e^{\lambda x}\left\{\partial_{t}+\partial_{x}^{3}\right\}\left(\mu_{R} w\right)\right\|_{L^{8 / 7}(\mathbb{R} \times[0,1])} \\
& +c_{0}\left\|e^{\lambda x}\left\{\partial_{t}+\partial_{x}^{3}\right\}\left(\mu_{R} w\right)\right\|_{L_{x}^{16 / 15} L_{t}^{16 / 11}(\mathbb{R} \times[0,1])} \\
& \leq c_{0}\left\|e^{\lambda x} V_{1} \partial_{x}\left(\mu_{R} w\right)\right\|_{L^{8 / 7}(\mathbb{R} \times[0,1])} \\
& +c_{0}\left\|e^{\lambda x} V_{1} \partial_{x}\left(\mu_{R} w\right)\right\|_{L_{x}^{16 / 15} L_{t}^{16 / 11}(\mathbb{R} \times[0,1])} \\
& +c_{0}\left\|e^{\lambda x} V_{2} \mu_{R} w\right\|_{L^{8 / 7}(\mathbb{R} \times[0,1])}+c_{0}\left\|e^{\lambda x} V_{2} \mu_{R} w\right\|_{L_{x}^{16 / 15} L_{t}^{16 / 11}(\mathbb{R} \times[0,1])} \\
& +c_{0}\left\|e^{\lambda x} F_{R}\right\|_{L^{8 / 7}(\mathbb{R} \times[0,1])}+c_{0}\left\|e^{\lambda x} F_{R}\right\|_{L_{x}^{16 / 15} L_{t}^{16 / 11}(\mathbb{R} \times[0,1])},
\end{aligned}
$$

where $c_{0}$ denotes the constant coming from Lemma 2.2, (2.7)-(2.8). From (2.63) we have for $R$ sufficiently large the following four estimates

$$
\begin{aligned}
& c_{0}\left\|e^{\lambda x} V_{1} \partial_{x}\left(\mu_{R} w\right)\right\|_{L^{8 / 7}(\mathbb{R} \times[0,1])} \\
& \leq c_{0}\left\|e^{\lambda x} \partial_{x}\left(\mu_{R} w\right)\right\|_{L_{x}^{16} L_{t}^{16 / 5}(\mathbb{R} \times[0,1])}\left\|V_{1}\right\|_{L_{x}^{16 / 13} L_{t}^{16 / 9}(\{x \geq R\} \times[0,1])} \\
& \leq \frac{1}{10}\left\|e^{\lambda x} \partial_{x}\left(\mu_{R} w\right)\right\|_{L_{x}^{16} L_{t}^{16 / 5}(\mathbb{R} \times[0,1])},
\end{aligned}
$$




$$
\begin{aligned}
& c_{0}\left\|e^{\lambda x} V_{1} \partial_{x}\left(\mu_{R} w\right)\right\|_{L_{x}^{16 / 15} L_{t}^{16 / 11}(\mathbb{R} \times[0,1])} \\
& \leq c_{0}\left\|e^{\lambda x} \partial_{x}\left(\mu_{R} w\right)\right\|_{L_{x}^{16} L_{t}^{16 / 5}(\mathbb{R} \times[0,1])}\left\|V_{1}\right\|_{L_{x}^{16 / 14} L_{t}^{16 / 6}(\{x \geq R\} \times[0,1])} \\
& \leq \frac{1}{10}\left\|e^{\lambda x} \partial_{x}\left(\mu_{R} w\right)\right\|_{L_{x}^{16} L_{t}^{16 / 5}(\mathbb{R} \times[0,1])}, \\
& \quad c_{0}\left\|e^{\lambda x} V_{2} \mu_{R} w\right\|_{L^{8 / 7}(\mathbb{R} \times[0,1])}\left\|V_{2}\right\|_{L^{4 / 3}(\{x \geq R\} \times[0,1])} \\
& \leq c_{0}\left\|e^{\lambda x} \mu_{R} w\right\|_{L^{8}(\mathbb{R} \times[0,1])}\left\|\frac{1}{10}\right\| e^{\lambda x} \mu_{R} w \|_{L^{8}(\mathbb{R} \times[0,1])}, \\
& \quad c_{0}\left\|e^{\lambda x} V_{2} \mu_{R} w\right\|_{L_{x}^{16 / 15} L_{t}^{16 / 11}(\mathbb{R} \times[0,1])} \\
& \leq c_{0}\left\|e^{\lambda x} \mu_{R} w\right\|_{L^{8}(\mathbb{R} \times[0,1])}\left\|V_{2}\right\|_{L_{x}^{16 / 13} L_{t}^{16 / 9}(\{x \geq R\} \times[0,1])} \\
& \leq \frac{1}{10}\left\|e^{\lambda x} \mu_{R} w\right\|_{L^{8}(\mathbb{R} \times[0,1])} .
\end{aligned}
$$

Inserting (2.67)-(2.70) into (2.66) one gets that

$$
\begin{aligned}
& \left\|e^{\lambda x} \mu_{R} w\right\|_{L^{8}(\mathbb{R} \times[0,1])}+\left\|e^{\lambda x} \partial_{x}\left(\mu_{R} w\right)\right\|_{L_{x}^{16} L_{t}^{16 / 5}(\mathbb{R} \times[0,1])} \\
& \leq 2 c_{0}\left\|e^{\lambda x} F_{R}\right\|_{L^{8 / 7}(\mathbb{R} \times[0,1])}+2 c_{0}\left\|e^{\lambda x} F_{R}\right\|_{L_{x}^{16 / 15} L_{t}^{16 / 11}(\mathbb{R} \times[0,1])} .
\end{aligned}
$$

To estimate the left hand side of (2.71) it suffices to consider one of the terms in $F_{R}$ (see $(2.65)$ ), say $F_{3}$, since the proofs for $F_{1}, F_{2}, F_{4}$ are similar. We recall that the supports of the $F_{j}$ 's are contained in the interval $[R, 2 R]$. Thus,

$$
\begin{aligned}
& 2 c_{0}\left\|e^{\lambda x} F_{3}\right\|_{L^{8 / 7}(\mathbb{R} \times[0,1])} \\
& \leq \frac{2 c_{0}}{R^{2}}\left(\int_{0}^{1} \int_{R}^{2 R} e^{8 \lambda x / 7}\left|\partial_{x} w(x, t)\right|^{8 / 7} d x d t\right)^{7 / 8} \\
& \leq \frac{2 c_{0}}{R^{2}} e^{2 \lambda R}\left(\int_{0}^{1} \int_{R}^{2 R}\left|\partial_{x} w(x, t)\right|^{8 / 7} d x d t\right)^{7 / 8} \leq \frac{2 c_{0}^{\prime}}{R^{2}} e^{2 \lambda R}
\end{aligned}
$$

and

$$
\begin{aligned}
& 2 c_{0}\left\|e^{\lambda x} F_{3}\right\|_{L_{x}^{16 / 15} L_{t}^{16 / 11}(\mathbb{R} \times[0,1])} \\
& \leq \frac{2 c_{0}}{R^{2}}\left(\int_{R}^{2 R} e^{16 \lambda x / 15}\left(\int_{0}^{1}\left|\partial_{x} w(x, t)\right|^{16 / 11} d t\right)^{11 / 15} d x\right)^{15 / 16} \\
& \leq \frac{2 c_{0}}{R^{2}} e^{2 \lambda R}\left(\int_{R}^{2 R}\left(\int_{0}^{1}\left|\partial_{x} w(x, t)\right|^{16 / 11} d t\right)^{11 / 15} d x\right)^{15 / 16} \\
& \leq \frac{2 c_{0}^{\prime}}{R^{2}} e^{2 \lambda R}
\end{aligned}
$$


where the constant $c_{0}^{\prime}$ in (2.72)-(2.73) is independent of $\lambda$. Since

$$
\left\|e^{\lambda x}\left(\mu_{R} w\right)\right\|_{L^{8}(\mathbb{R} \times[0,1])} \geq\left(\int_{0}^{1} \int_{x>2 R} e^{8 \lambda x}|w(x, t)|^{8} d x d t\right)^{1 / 8},
$$

combining (2.71)-(2.73) we conclude that

$$
\left(\int_{0}^{1} \int_{x>2 R} e^{8 \lambda(x-2 R)}|w(x, t)|^{8} d x d t\right)^{1 / 8} \leq \frac{4 c_{0}^{\prime}}{R^{2}} .
$$

Now letting $\lambda \uparrow \infty$ it follows that

$$
w(x, t) \equiv 0 \quad \text { for } \quad x>2 R, t \in[0,1],
$$

which combined with Theorem 2.4 yields the proof.

\section{$\S 3$. Sketch of the proof of Theorem 1 in the general case.}

In this section we shall comment on the modifications needed for the proof in the previous section to treat the general equation in (1.6).

Taking the $x$-derivative of order $k$, with $k=1,2$ of the equation (1.6) and using the notation

$$
w_{j}(x, t)=\partial_{x}^{j}\left(u_{1}-u_{2}\right)(x, t), \quad j=0,1,2,
$$

we obtain the system (written in a convenient form)

$$
\left\{\begin{array}{l}
\partial_{t} w_{0}+\partial_{x}^{3} w_{0}+\sum_{j=0}^{2} a_{0, j}(x, t) w_{j}=0 \\
\partial_{t} w_{1}+\partial_{x}^{3} w_{1}+a_{1,0}(x, t) \partial_{x} w_{2}+\sum_{j=0}^{2} a_{1, j+1}(x, t) w_{j}=0 \\
\partial_{t} w_{2}+\partial_{x}^{3} w_{2}+a_{2,0}(\cdot) \partial_{x}^{2} w_{2}+a_{2,1}(\cdot) \partial_{x} w_{2}+\sum_{j=0}^{2} a_{2, j+2}(\cdot) w_{j}=0
\end{array}\right.
$$

where the coefficients $a_{j, l}$ 's depend on $u_{1}, u_{2}$ and its derivatives up to order four.

Our goal is to remove the term $a_{2,0}(x, t) \partial_{x}^{2} w_{2}$ in the last equation in (3.2). Thus, following the idea of Hayashi and Ozawa in [1] we first introduce the new variable (an integrating factor)

$$
\tilde{w}_{2}(x, t)=e^{\frac{1}{3} \int_{0}^{x} a_{2,0}(s, t) d s} w_{2}(x, t)=e^{\phi(x, t)} w_{2}(x, t) .
$$

Next, we multiply the last equation in (3.2) by $e^{\phi}$ and use that $w_{2}=e^{-\phi} \tilde{w}_{2}$ to rewrite the system (3.2) in terms of the new unknown functions $\left(w_{0}, w_{1}, \tilde{w}_{2}\right)$. This new system is a diagonal one and does not contain terms involving the second derivatives of the functions $\left(w_{0}, w_{1}, \tilde{w}_{2}\right)$ so that the argument provided in the previous section can be applied yielding the desired result. 


\section{References}

[1] Hayashi, N., and Ozawa, T., Remarks on nonlinear Schrödinger equations in one space dimension, Diff. \& Integral Eqs. 7 (1994), 453-461.

[2] Kato, T., On the Cauchy problem for the (generalized) Korteweg-de Vries equation, Advances in Mathematics Supplementary Studies, Studies in Applied Math. 8 (1983), 93-128.

[3] Kenig, C. E., Ponce, G., and Vega, L., Well-posedness and scattering results for the generalized Korteweg-de Vries equation via the contraction principle, Comm. Pure Appl. Math. 46 (1993), 527-620.

[4] Higher-order nonlinear dispersive equations, Proc. Amer. Math. Soc. 122 (1994), 157-166.

[5] - On the support of solutions to the generalized KdV equation, Annales de l'I.H.P. Analyse Non Linéaire 19 (2002), 191-208.

[6] - On unique continuation for the nonlinear Schrödinger equations, Comm. Pure Appl. Math., 60, (2002), 1247-1262.

[7] Kenig, C. E., Ruiz, A., and Sogge, C., Uniform Sobolev inequalities and unique continuation for second order constant coefficient differential operators, Duke Math. J. 55 (1987), 329-347.

[8] Kenig, C. E., and Sogge, C., A note on unique continuation for Schrödinger's operator, Proc. Amer. Math. Soc. 103 (1988), 543-546.

[9] Korteweg, D. J., and de Vries, G., On the change of form of long waves advancing in a rectangular canal, and on a new type of long stationary waves, Philos. Mag. 539 (1895), $422-443$.

[10] Kruzhkov, S. N., and Faminskii, A. V., Generalized solutions of the Cauchy problem for the Korteweg-de Vries equation, Math. U.S.S.R. Sbornik 48 (1984), 93-138.

[11] Saut, J.-C., and Scheurer, B., Unique continuation for some evolution equations, J. Diff. Eqs. 66 (1987), 118-139.

[12] Stein, E. M., Harmonic Analysis, Princeton University Press (1993).

[13] Zhang, B.-Y., Unique continuation for the Korteweg-de Vries equation, SIAM J. Math. Anal. 23 (1992), 55-71.

Department of Mathematics, University of Chichgo, Chicago, IL 60637

E-mail address: cek@math.uchicago.edu

Department of Mathematics, University of California, Santa Barbara, CA 93106 E-mail address: ponce@math.ucsb.edu

Department of Mathematics, Universidad del Pais Vasco, Apartado 644, 48080 BILBAO, SPAIN

E-mail address: mtpvegol@lg.ehu.es 